\title{
Allergy to topical corticosteroids in inflammatory bowel disease
}

\section{CASE REPORT}

A 57 year old Caucasian man presented with an eight week history of loose bowel motions 10 times daily, with blood, slime, and mucus. Sigmoidoscopy showed an abnormal vascular pattern and a granular mucosa. Rectal biopsy was reported histologically to show inflammatory bowel disease, and treatment was initiated with oral Asacol (mesalazine) $800 \mathrm{mg}$ three times daily and Predfoam (prednisolone metasulphobenzoate sodium) enema twice daily. This treatment was continued over a period of about eight weeks, initially with some improvement but latterly relapsing to the pretreatment state Fibreoptic colonoscopy was then performed, revealing inflammatory changes up to the distal sigmoid colon. Biopsies were suggestive of Crohn's disease. Treatment was continued for a further three months without improvement. The patient decided on his own initiative to stop the Predfoam enemas but continued on Asacol. He reported four weeks later that within 48 hours of stopping Predfoam his symptoms had resolved entirely.

In view of the history of failure to respond to apparently appropriate treatment with a topical steroid, and the improvement on withdrawal, the theoretical possibility of "contact allergy" was entertained, and he was referred for patch testing. He gave no history of allergy, nor of use of topical or inhaled steroids. He was tested with a standard battery of contact allergens, including tixocortol pivalate and budesonide. At 72 hours he demonstrated a ++ positive reaction to budesonide. At follow up three months later he remained symptom free. It was not considered ethical or clinically indicated to rechallenge the patient, and he declined further investigation.

\section{DISCUSSION}

Topical steroids are now recognised to be common allergens in the skin. ${ }^{1}$ It is standard practice to test all patients with suspected contact dermatitis for steroid allergy. Tixocortol pivalate and budesonide are regarded as markers for the great majority of reactions to topical steroids, and a positive reaction to one of these markers is not regarded as indicating allergy to that steroid alone. ${ }^{2}{ }^{3}$ Topical steroids are currently the commonest medicaments to cause contact dermatitis. $^{4}$

In patients with inflammatory bowel disease it is less easy to investigate for possible allergic reactions on the bowel mucosa, and the subject has been investigated relatively little. In this case, the history of failure of the inflammatory bowel disease to respond to appropriate topical steroid therapy, and to resolve on withdrawal of the steroid, was thought clinically to be reminiscent of the pattern of clinical presentation of contact allergy to topical steroid in patients with eczema. In patients with contact sensitisation in the skin, the presence of an already damaged and inflamed skin appears to increase the penetration of potential contact allergens, and development of sensitisation. It could be postulated that an analogous situation arises in patients with inflammatory bowel disease of the distal bowel using long or medium term rectal steroids.

It is possible that the history in this patient, with failure to improve with the use of a topical steroid and improvement on withdrawal, and the positive patch test reaction to budesonide were all coincidental. However, if the analogy to the situation with patients with inflammatory skin disease holds true, this may be clinically important. This scenario has not to our knowledge been previously reported in patients using topical rectal steroids. Delayed hypersensitivity reactions to steroids in nasal sprays has been reported, as has a case of allergic contact stomatitis. ${ }^{5}$ Reaction to systemic administration of steroids has also been occasionally described. ${ }^{6}$ We have no idea whether our patient is unique or whether this phenomenon may be common but previously unrecognised. It must be remembered that topical steroids were in use for nearly 40 years before contact allergy became widely recognised. Allergy to topical steroids should be considered in patients using rectal steroid preparations whose condition shows unexpected failure to improve or unexpected deterioration.

B E Monk, Department of Dermatology, Cranfield Postgraduate Medical School at Bedford, Bedford Hospital NHS Trust, Bedford MK42 9D, UK

D Skipper,

Department of Surgery, Cranfield Postgradua Medical School at Bedford Bedford Hospital NHS Trust, Bedford MK42 9DJ, UK

Correspondence to: B E Monk ; barry@bmonk4.fsnet.co.uk

\section{REFERENCES}

1 Dooms-Goossens AE, Degreef HJ, Marien $\mathrm{KJ}$, et al. Contact allergy to corticosteroids: a frequently missed diagnosis. J Am Acad Dermatol 1989.21.538-43.

2 English JS. Corticosteroid-induced dermatitis: a pragmatic approach. Clin Exp Dermatol 2000;25:261-4.

3 Boffa MJ, Wilkinson SM, Beck MH. Screening for corticosteroids allergy. Contact Dermatitis 1995:33:149-51.

4 Monk BE. Patch testing in a British district general hospital. Clin Exp Dermatol 999.24:484-6.

5 Bircher AJ, Pelloni F, Langauer MS, et al. Delayed hypersensitivity to corticosteroids applied to mucous membranes. Br J Dermatol 1996;135:310-3

6 Pollock B, Wilkinson SM, MacDonald Hull SP. Chronic urticaria associated with intra-articular methylprednisolone $\mathrm{Br} J$ Dermatol 2001:144:1228-30. 